\title{
Residual flow in false lumen of chronic descending aortic dissection
}

\author{
M. Boulaksil ${ }^{1}$ (D) - S. S. Liem ${ }^{1}$ M. Akarkach ${ }^{1}$ J. Timmermans ${ }^{1}$
}

Published online: 9 November 2017

(c) The Author(s) 2017. This article is an open access publication.

A 58-year-old male patient has a history of a Stanford type A aortic dissection running up to the left common iliac artery for which a composite aortic valve graft replacement was performed approximately three years ago. This time, the patient presented with fever and chills. We performed a transesophageal echocardiography (TEE), which excluded vegetations.

Upon retracting the TEE probe, the descending aorta showed two compartments (Fig. 1a) separated by a dissected intimal layer (Fig. 1a, arrow heads); the largest compartment is the false lumen (Fig. 1a, hash). Colour Doppler imaging showed laminar flow through the true aortic lumen (Fig. 1a, asterisk). In the false aortic lumen, slow rotating blood flow existed (online video). This phenomenon was not present more proximally in the descending aorta at the aortic arch (Fig. 1b). Residual blood flow may persist in the false lumen years after aortic dissection because of multiple fenestrations in the dissected intimal layer providing entry and exit locations for blood flow. In approximately $70 \%$ of patients with acute type A aortic dissection, the dissection extends beyond the ascending aorta [1-3]. After repair, these patients show an increased risk of developing postdissection aortic aneurysm mainly through false lumen di-

Electronic supplementary material The online version of this article (https://doi.org/10.1007/s12471-017-1052-6) contains supplementary material, which is available to authorized users.

M. Boulaksil

m.boulaksil@gmail.com

1 Department of Cardiology, Radboud University Medical

Center, Nijmegen, The Netherlands latation, requiring late distal aortic re-interventions in up to one-fifth of cases $[3,4]$. False lumen patency appeared to be a major risk factor for late re-intervention and was associated with an accelerated annual growth rate [3-5]. Therefore, long-term follow-up and close surveillance of these patients are imperative.

Open Access This article is distributed under the terms of the Creative Commons Attribution 4.0 International License (http:// creativecommons.org/licenses/by/4.0/), which permits unrestricted use, distribution, and reproduction in any medium, provided you give appropriate credit to the original author(s) and the source, provide a link to the Creative Commons license, and indicate if changes were made.

\section{References}

1. Conzelmann LO, Weigang E, Mehlhorn U, et al. Mortality in patients with acute aortic dissection type A: analysis of pre- and intraoperative risk factors from the German Registry for Acute Aortic Dissection Type A (GERAADA). Eur J Cardiothorac Surg. 2016;49:e44-52.

2. Rylski B, Hahn N, Beyersdorf F, et al. Fate of the dissected aortic arch after ascending replacement in type A aortic dissection. Eur J Cardiothorac Surg. 2017;51:1127-34.

3. Kimura N, Tanaka M, Kawahito K, et al. Influence of patent false lumen on long-term outcome after surgery for acute type A aortic dissection. J Thorac Cardiovasc Surg. 2008;136:1160-6.

4. Fattouch K, Sampognaro R, Navarra E, et al. Long-term results after repair of type A acute aortic dissection according to false lumen patency. Ann Thorac Surg. 2009;88:1244-50.

5. Halstead JC, Meier M, Etz C, et al. The fate of the distal aorta after repair of acute type A aortic dissection. J Thorac Cardiovasc Surg. 2007;133:127-35. 

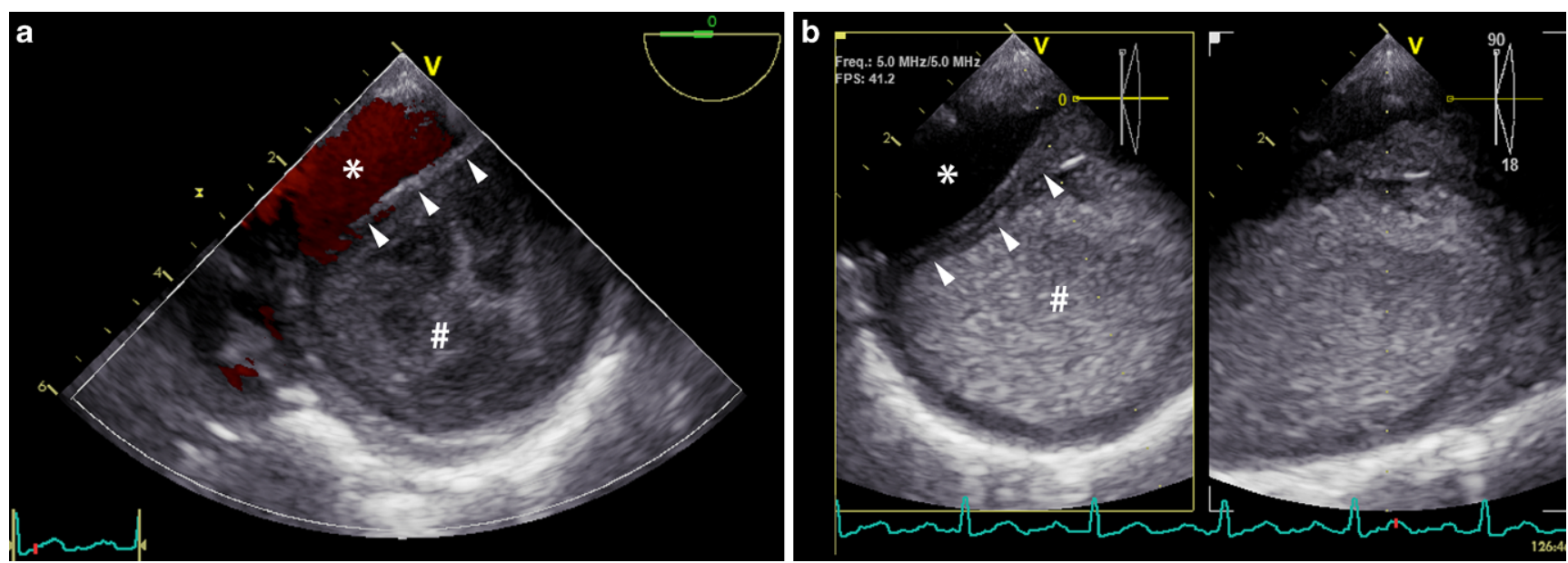

Fig. 1 Transverse cross-sectional views of the descending aorta at different levels as documented with TEE. a Transversal cross section of the descending aorta showing two compartments separated by a dissected intimal layer (arrow heads). The largest compartment is the false lumen (hash). Colour Doppler showed laminar flow through the true aortic lumen (asterisk) and no entry site to the false lumen. In the false lumen, slow rotating blood flow existed. See also online video. b Transversal cross section of the aorta more proximally at the aortic arch. Here, slow rotating blood flow in the false lumen was not observed. Asterisk true aortic lumen, hash false aortic lumen, arrow heads dissection layer 